\title{
SUB-KILOPARSEC ALMA IMAGING OF COMPACT STAR-FORMING GALAXIES AT $z \sim 2.5$ : REVEALING THE FORMATION OF DENSE GALACTIC CORES IN THE PROGENITORS OF COMPACT QUIESCENT GALAXIES
}

\author{
G. Barro ${ }^{1}$, M. Kriek ${ }^{1}$, P. G. Pérez-González ${ }^{2}$, J. R. Trump ${ }^{3,12}$, D. C. $\mathrm{KoO}^{4}$, S. M. Faber ${ }^{4}$, A. Dekel ${ }^{5}$, J. R. Primack ${ }^{6}$, \\ Y. Guo ${ }^{3}$, D. D. Kocevski ${ }^{7}$, J. C. Muñoz-Mateos ${ }^{8}$, W. Rujoparkarn ${ }^{9,10}$, and K. Seth ${ }^{11}$ \\ ${ }^{1}$ University of California, Berkeley, Berkeley, CA, USA \\ ${ }^{2}$ Universidad Complutense de Madrid, Madrid, Spain \\ ${ }^{3}$ Pennsylvania State University, University Park, PA, USA \\ ${ }^{4}$ University of California, Santa Cruz, Santa Cruz, CA, USA \\ ${ }^{5}$ The Hebrew University, Jerusalem, Israel \\ ${ }^{6}$ Santa Cruz Institute for Particle Physics, Santa Cruz, CA, USA \\ ${ }^{7}$ Colby College, Waterville, ME, USA \\ ${ }^{8}$ European Southern Observatory, Casilla, Santiago, Chile \\ ${ }^{9}$ Kavli Institute for the Physics of the universe \\ ${ }^{10}$ Chulalongkorn University, Pathumwan, Bangkok, Thailand \\ ${ }^{11}$ North American ALMA Science Center, National Radio Astronomy Observatory, Charlottesville, VA, USA \\ Received 2016 June 3; revised 2016 June 27; accepted 2016 June 28; published 2016 August 16
}

\begin{abstract}
We present spatially resolved Atacama Large Millimeter/submillimeter Array (ALMA) $870 \mu$ m dust continuum maps of six massive, compact, dusty star-forming galaxies at $z \sim 2.5$. These galaxies are selected for their small rest-frame optical sizes $\left(r_{\mathrm{e}, \mathrm{F} 160 \mathrm{~W}} \sim 1.6 \mathrm{kpc}\right)$ and high stellar mass densities that suggest that they are direct progenitors of compact quiescent galaxies at $z \sim 2$. The deep observations yield high far-infrared (FIR) luminosities of $L_{\mathrm{IR}}=10^{12.3-12.8} L_{\odot}$ and star formation rates (SFRs) of SFR $=200-700 M_{\odot} \mathrm{yr}^{-1}$, consistent with those of typical star-forming "main sequence" galaxies. The high spatial resolution (FWHM $\sim 0$ ". 12-0". 18) ALMA and Hubble Space Telescope photometry are combined to construct deconvolved, mean radial profiles of their stellar mass and (UV+IR) SFR. We find that the dusty, nuclear IR-SFR overwhelmingly dominates the bolometric SFR up to $r \sim 5 \mathrm{kpc}$, by a factor of over $100 \times$ from the unobscured UV-SFR. Furthermore, the effective radius of the mean SFR profile $\left(r_{\mathrm{e}, \mathrm{SFR}} \sim 1 \mathrm{kpc}\right)$ is $\sim 30 \%$ smaller than that of the stellar mass profile. The implied structural evolution, if such nuclear starburst last for the estimated gas depletion time of $\Delta t= \pm 100 \mathrm{Myr}$, is a $4 \times$ increase of the stellar mass density within the central $1 \mathrm{kpc}$ and a $1.6 \times$ decrease of the half-mass-radius. This structural evolution fully supports dissipation-driven, formation scenarios in which strong nuclear starbursts transform larger, star-forming progenitors into compact quiescent galaxies.
\end{abstract}

Key words: galaxies: evolution - galaxies: high-redshift - galaxies: photometry

\section{INTRODUCTION}

The majority of star-forming galaxies (SFGs) follow a relatively tight, almost linear relation between star formation rate (SFR) and stellar mass, usually referred to as the star formation "main sequence" that seems to be in place since $z \sim 5-6$ (SF-MS; e.g., Noeske et al. 2007; Whitaker et al. 2012). The ubiquitous and tight SF-MS suggests that the majority of the stars are formed in a predominantly smooth, secular mode. Furthermore, there is also evidence that, despite their wide range of sizes and morphologies, most of the stars in SFGs are formed in disks which are growing from the inside out, thus increasing their sizes with cosmic time (Nelson et al. 2013, 2015; Wuyts et al. 2013). The progressive structural growth in the SF-MS is consistent with the classic notion of galaxy formation in a $\Lambda \mathrm{CDM}$ universe in which gas accreted from dark matter halos cools and forms new stars in disks with increasingly larger-scale lengths with cosmic time (e.g., Fall \& Efstathiou 1980; Mo et al. 1998).

A challenge to this simplified picture are the small sizes $\left(r_{e} \sim 1 \mathrm{kpc}\right)$ of the first massive quiescent galaxies at $z \gtrsim 1.5-3$ (e.g., van der Wel et al. 2014 and references therein). On one hand, their small sizes might be the consequence of having

\footnotetext{
${ }^{12}$ Hubble Fellow.
}

smaller star-forming progenitors formed at earlier times when the universe was more dense (i.e., more concentrated halos and higher gas fractions). On the other hand, compact quiescent galaxies could form in strongly dissipative processes, triggered by mergers or interaction-driven disk instabilities that cause a substantial growth of the nuclear stellar density as a result of gas-rich starbursts (Hopkins et al. 2008; Dekel et al. 2009). Both scenarios imply the formation of compact SFGs as the last stage before quenching star formation, but the predictions differ on whether these compact SFGs would exhibit extended SFR profiles, driving the inside-out size growth, or compact starforming regions (starbursts) triggered by the dissipative phase.

Such compact SFGs have been identified in sizable numbers and their small stellar sizes, steep mass profiles, and obscured SFR properties have been confirmed by multiple studies (Barro et al. 2013, 2014; van Dokkum et al. 2015). However, direct measurements of their spatial distribution of the star formation relative to the mass profile, needed to discriminate between the two formation scenarios discussed above, are still inconclusive. These measurements have proven very difficult because even spatially resolved UV and optical SFR indicators based on Hubble Space Telescope (HST) observations are significantly affected by the high dust obscuration, particularly in galaxy centers (Wuyts et al. 2012; Tacchella et al. 2015), and farinfrared (FIR) observations, sensitive to ionizing radiation re- 

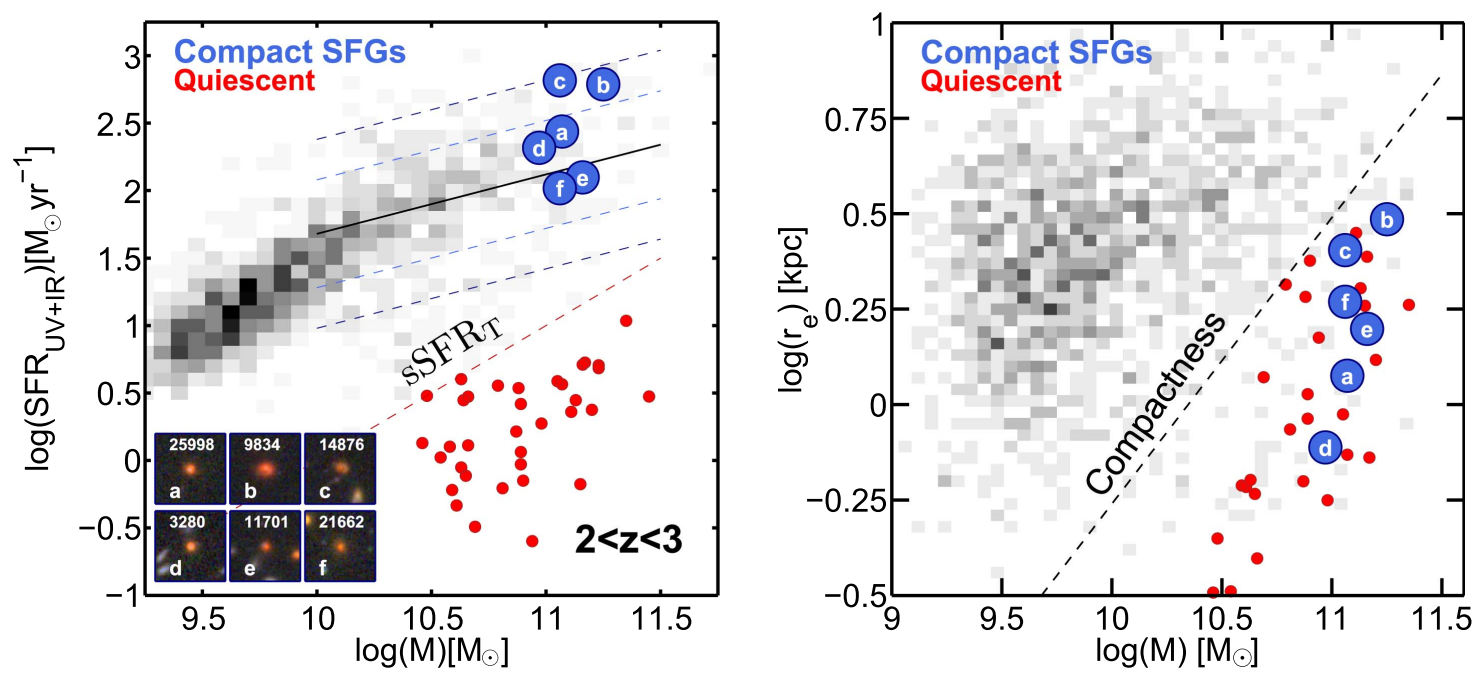

Figure 1. Left: SFR-mass diagram for galaxies in CANDELS GOODS-S at $2<z<3$. The grayscale density bins map the location of the SFR-MS. The solid black and dashed blue lines depict the best fit and $2.5 \times$ and $5 \times$ limits above and below the SFR-MS. The blue circles depict the compact SFGs observed with ALMA. The subpanels in the bottom left corner show the $5^{\prime \prime} \times 5^{\prime \prime}$ ACS/WFC3 $z J H$ images of the ALMA galaxies. The red dashed line marks the threshold in sSFR (log(sSFR/ $\left.\mathrm{Gyr}^{-1}\right)<-1$ ) used to identify quiescent galaxies (red circles). Right: mass-size distribution for the same galaxies as in the left panel. The dashed line marks the compactness threshold, $\log \left(\Sigma_{1.5}\right)=10.4 M_{\odot} \mathrm{kpc}^{-1.5}$.

emitted by the dust, usually have very poor spatial resolution. Modern (sub)millimeter/radio interferometers such as Atacama Large Millimeter/submillimeter Array (ALMA) and JVLA have opened a new window into this regime and enable us to measure the dust emission with high sensitivity and similar spatial resolution as those from $H S T$ observations.

Here, we exploit a joint analysis of the high spatial resolution $H S T / \mathrm{ACS}$ and WFC3 and ALMA continuum imaging to simultaneously characterize the UV- and IR-SFR profiles and the stellar mass profiles of 6 compact SFGs at $z \sim 2.5$. Throughout this Letter, we quote magnitudes in the AB system, assume a Chabrier (2003) initial mass function, and adopt the following cosmological parameters: $\left(\Omega_{M}, \Omega_{\Lambda}, h\right)=(0.3$, $0.7,0.7)$.

\section{DATA AND SAMPLE SELECTION}

The six galaxies analyzed in this Letter are drawn from the sample of compact SFGs in the CANDELS (Grogin et al. 2011) GOODS-S region presented in Barro et al. (2014). The UV to near-IR spectral energy distributions (SEDs) include extensive multi-band data ranging from $U$ to $8 \mu \mathrm{m}$ (Guo et al. 2013). Furthermore, we include Spitzer/MIPS 24 and $70 \mu \mathrm{m}$ data $(30 \mu \mathrm{Jy}$ and $1 \mathrm{mJy}, 5 \sigma)$ from Pérez-González et al. (2008), and PACS 70, 100, and $160 \mu \mathrm{m}(0.7 \mathrm{mJy}, 5 \sigma)$, and SPIRE 250, 350, and $500 \mu \mathrm{m}(1 \mathrm{mJy}, 5 \sigma)$ from the GOODS-Herschel (Elbaz et al. 2011) and PEP (Magnelli et al. 2013) surveys.

The compact SFGs were identified following the method described in Barro et al. $(2013,2014)$. Briefly, we require galaxies to be massive $\left(\log \left(M / M_{\odot}\right)>10.5\right)$ and star-forming $\left(\log \left(\mathrm{sSFR} / \mathrm{Gyr}^{-1}\right)>-1\right)$, and we impose a compactness criterion (Barro et al. 2013), $\log \left(M / \pi r_{\mathrm{e}}^{1.5}\right)=10.4 M_{\odot} \mathrm{kpc}^{-1.5}$, to identify galaxies with similar structural properties as quiescent galaxies at that redshift. Finally, we choose FIR bright galaxies detected in Spitzer and Herschel with predicted ALMA $870 \mu \mathrm{m}$ fluxes above $\sim 1 \mathrm{mJy}$. Figure 1 illustrates the selection criteria by showing the location of the compact SFGs (blue circles) observed with ALMA overlaid in the SFR-mass and mass-size diagrams for galaxies more massive than $\log (M /$ $\left.M_{\odot}\right)>9$ at $2<z<3$ in the CANDELS GOODS-S catalog.

The submillimeter observations of the six targets were taken as part of an ALMA cycle-2 campaign (ID: 2013.1.00576.S; PI: G. Barro) aimed at studying the dust emission continuum in compact SFGs at $z=2-3$. The observations were carried out on 2015 August 29 and 2015 September 7 in band 7 using four spectral windows in the largest bandwidth mode. The onsource integration time was $1800 \mathrm{~s}$ in the longest array configuration, C34-7. Flux, phase, and bandpass calibrators were also obtained, for a total time of $\sim 3 \mathrm{hr}$. We used the CASA software to process and clean the data. The cleaning algorithm was run using a natural weighting for the uv visibility plane. The average angular resolution of the observations is FWHM $=0$ !" $14 \times 0$ !" 11 , with a major-axis position angle ranging from $3^{\circ}$ to $65^{\circ}$ (see Figure 3 ). The rms noise of the observations is $\sigma=40 \mu \mathrm{Jy}_{\text {beam }}{ }^{-1}$ or $2.4 \mathrm{mJy} \operatorname{arcsec}^{-2}$. The depth of the ALMA observations allows reliable measurements of the surface brightness profile at a $3 \sigma$ level down to a radius of at least $5 \times$ the HWHM of the ALMA clean beam. We find and correct an average systematic offset between the HST and ALMA astrometry ${ }^{13}$ of $\triangle$ R.A. $=-0 . " 08$ and $\Delta$ decl. $=0$ ". 27 with an rms $\sim 0$ ". 06 .

\section{OPTICAL/NIR AND MIR/SUBMILLIMETER SED FITS: $M_{\star}, \mathrm{SFR}, M_{\text {dust }}$, AND $M_{\text {gas }}$}

\subsection{Models and Assumptions}

We fit the optical/NIR SEDs to calculate stellar masses using FAST (Kriek et al. 2009) and assuming Bruzual \& Charlot (2003) stellar population synthesis models, and the Calzetti et al. (2000) dust law with attenuation $0<A_{V}<4$ (see Figure 2). We also assume an exponentially declining star formation history with timescale $\tau$ and age $t$ (see Santini et al. 2015 for more details).

\footnotetext{
${ }_{13}$ The offsets are consistent with recent results from a JVLA survey of GOODS-S (W. Rujopakarn 2016, in preparation).
} 

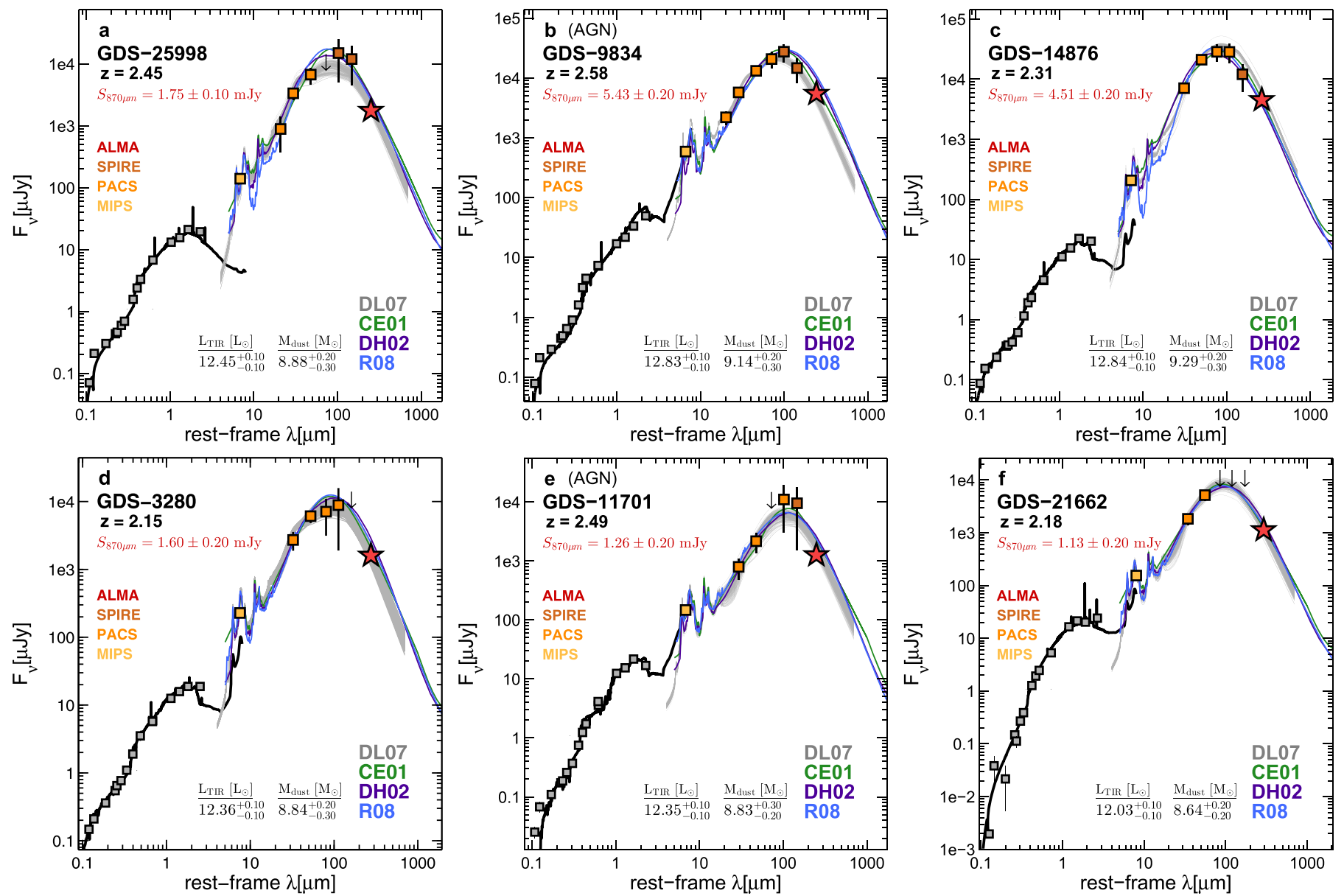

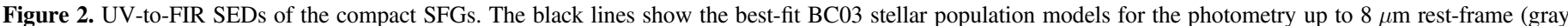

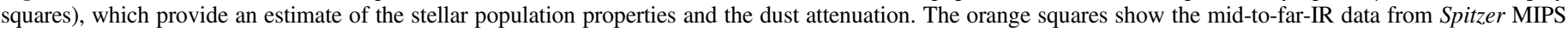

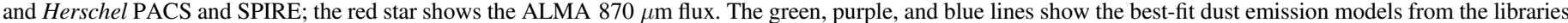

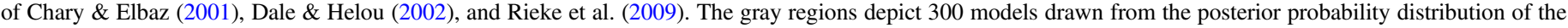
emcee fit to the Draine \& Li (2007) models. The median values and confidence intervals for $L_{\mathrm{TIR}}$ and $M_{\mathrm{dust}}$ are indicated.

We fit the mid-to-FIR SEDs to the dust emission templates of Chary \& Elbaz (2001), Dale \& Helou (2002), and Rieke et al. (2009). Moreover, we fit the models by Draine \& Li (2007, hereafter DL07) to estimate the physical properties of the dust. In these models, dust is exposed to a range of starlight intensities indicated by a scale factor $U$. The majority of the dust is heated by a constant intensity $U_{\min }$, while a smaller fraction $\gamma$ of the dust is exposed to variable intensities ranging from $U_{\min }$ to $U_{\max }$. The parameter, $q$, controls the fraction of the dust grains in the form of polycyclic aromatic hydrocarbon grains. We find the best-fit DL07 models and the corresponding confidence intervals by exploring the parameter space using the Python Markov Chain Monte Carlo package emcee (ForemanMackey et al. 2013).

We also estimate the molecular gas content using the gas-todust ratio by assuming $\delta_{\mathrm{GDR}} M_{\mathrm{dust}}=M_{\mathrm{gas}}$. The value of $\delta_{\mathrm{GDR}}$ depends primarily on the metallicity of the galaxy (e.g., Sandstrom et al. 2013). We estimate $\delta_{\mathrm{GDR}}(Z)$ using the massmetallicity (MZR) relation at $z \sim 2$ of Steidel et al. (2014) and the empirical calibration of Magdis et al. (2012). For the relatively small range of galaxy masses in our sample, the average metallicity is $Z=12+\log (\mathrm{O} / \mathrm{H})=8.57$, which implies gas-to-dust ratios of $\delta_{\mathrm{GDR}} \sim 100$.

We compute the total SFR by adding the unobscured and obscured star formation, traced by the UV and IR emission, respectively, following Kennicutt (1998; see also Bell et al. 2005):

$$
\mathrm{SFR}_{\mathrm{UV}+\mathrm{IR}}=1.09 \times 10^{-10}\left(L_{\mathrm{IR}}+3.3 L_{2800}\right)\left[M_{\odot} \mathrm{yr}^{-1}\right]
$$

where $L_{\mathrm{IR}}$ is the total IR luminosity $\left(L_{\mathrm{IR}} \equiv L(8-1000 \mu \mathrm{m})\right)$ derived from the average value of the best-fit templates to the four dust emission libraries, and $L_{2800}=\nu L_{\nu}(2800)$ is estimated from the best-fit SED models.

\subsection{Integrated IR-SFRs, UV-SFRs, Dust, and Gas Masses}

The compact SFGs exhibit high IR luminosities ranging from $L_{\mathrm{IR}}=10^{12.03-12.80} L_{\odot}$ and SFR $=150-730 M_{\odot} \mathrm{yr}^{-1}$ that straddle the SFR main sequence at $\log \left(M / M_{\odot}\right) \sim 11$ (Figure 1). Two of the galaxies have slightly larger SFRs than the median of SFR $\sim 300 M_{\odot} \mathrm{yr}^{-1}$, yet still within $5 \times$ of the SF-MS. The total SFR is strongly dominated by the IR emission. The average ratio of integrated $\mathrm{SFR}_{\mathrm{IR}} / \mathrm{SFR}_{\mathrm{UV}}=70-100$ implies optical attenuations of $A_{\mathrm{V}} \gtrsim 2 \mathrm{mag}$. However, the values determined from SED fitting are only $A_{\mathrm{V}} \sim 1.3-1.6 \mathrm{mag}$.

The IR-SFRs determined from the different dust template libraries are in excellent agreement, with a median difference and $1 \sigma$ scatter of $\triangle \mathrm{SFR}=0.01 \pm 0.13 \mathrm{dex}$. The SFRs are also consistent with estimates based on the MIPS $24 \mu \mathrm{m}$ flux alone, using the empirical relation from Wuyts et al. (2011), 

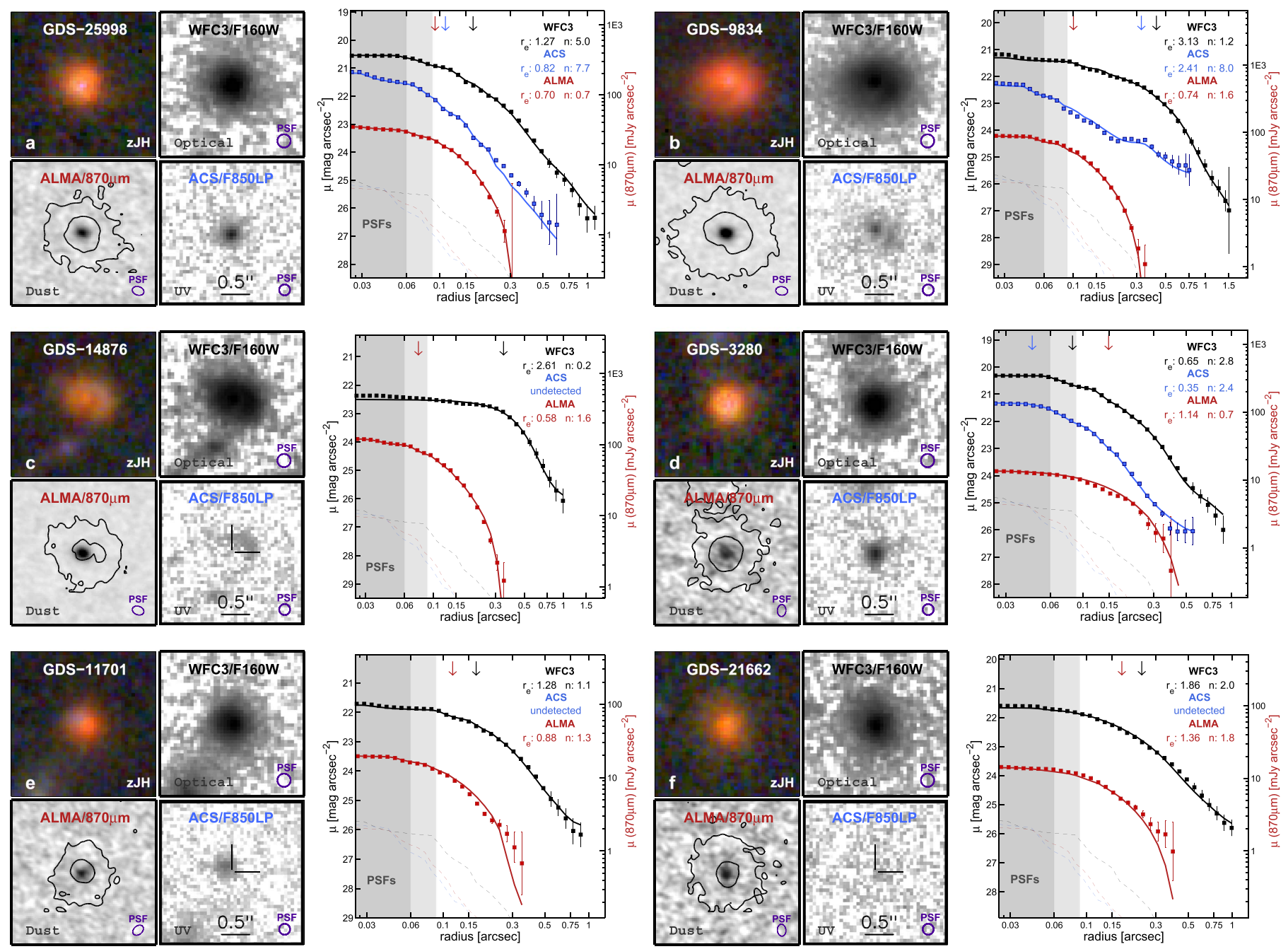

Figure 3. 2" $5 \times 2$ " 5 images and surface brightness profiles (un-corrected for the PSF) of the compact SFGs in ACS/F850LP, WFC3/F160W, and ALMA $870 \mu \mathrm{m}$ (with the F160W contours shown in black). The surface brightness profiles (squares) are measured along concentric ellipses that follow the geometry of the best-fit Sérsic model (solid lines). The ALMA $870 \mu \mathrm{m}$ profiles are scaled down arbitrarily with respect to the HST data (see right $y$-axis). The ALMA and ACS images have similar spatial resolution (FHWM $\sim 0$." 12) and are slightly smaller than for WFC3 (FHWM $\sim 0$ ". 18). The dashed lines show the PSF profiles and the shaded regions show the extent of their HWHM in F850LP and ALMA (dark gray) and F160W (light gray). The deconvolved GALFIT effective radii in kiloparsecs are indicated with arrows. The profiles are shown up to the radius where the errors become significant, typically $\sim 1^{\prime \prime}$ in F160W and $\sim 0$ ". 4 in ALMA.

$\Delta \mathrm{SFR}=0.02 \pm 0.26$ dex. The two X-ray-detected active galactic nuclei (AGNs) in the sample (GDS-9834 and GDS11701) exhibit slightly higher MIPS $24 \mu \mathrm{m}$ SFRs, likely as a result of hot dust emission from the AGNs at shorter wavelengths (see also Barro et al. 2014). We find similar SFR values when the $24 \mu \mathrm{m}$ flux is excluded from the fits.

The dust masses range from $M_{\text {dust }}=10^{8.05-9.29} M_{\odot}$ and are larger for the two galaxies with the highest SFRs. The gas masses determined from $\delta_{\mathrm{GDR}}$ and the MZR relation indicate gas fractions of the order of $f_{\text {gas }}=M_{\text {gas }} /\left(M_{\text {gas }}+M_{\star}\right)=$ $0.47_{-0.15}^{+0.19}$, consistent with previous works (Tacconi et al. 2010). The average depletion time, assuming no further gas replenishment, is relatively short, $t_{\mathrm{dpl}}=M_{\mathrm{gas}} / \mathrm{SFR}=230_{-120}^{+90} \mathrm{Myr}$.

\section{STRUCTURAL PROPERTIES}

\subsection{UV, Optical, and FIR Surface Brightness Profiles}

Figure 3 shows the images and surface brightness profiles of the galaxies in WFC3/F160W, ACS/F850LP, and ALMA $870 \mu \mathrm{m}$. At $z \sim 2.5$, these bands probe the rest-frame
UV, optical, and FIR, respectively. We account for the different spatial resolution of each data set by modeling the shape of the two-dimensional surface brightness profiles using GALFIT (Peng et al. 2002). The half-light radii and Sérsic indices, $n$, are determined using a single component fit. For the HST images, the PSFs are created with TinyTim (Krist 1995) as detailed in van der Wel et al. (2012). For ALMA, we used the synthetic PSF generated by CASA. The depth of the ALMA observations $\left(t_{\exp } \sim 30\right.$ minutes) provides a smooth, uniform coverage of the PSF. Figure 3 indicates the best-fit $r_{\mathrm{e}}$ (arrows) and $n$ in each band.

The mean radius containing $95 \%$ of the light from the observed FIR profiles is $\sim 4 \times$ smaller than that of the optical profile. The deconvolved, FIR profiles are also more compact than the optical in 5/6 galaxies. The exception is GDS-3280, which has the smallest optical size and a high $n$, while the morphology in ALMA exhibits some slight asymmetries. The ratio of the mean effective radii is $\left\langle r_{\mathrm{e}, \mathrm{F} 160 \mathrm{~W}}\right\rangle /\left\langle r_{\mathrm{e}, 870 \mu \mathrm{m}}\right\rangle=1.9$. Despite being more compact, the FIR profiles have, on average, lower (disk-like) Sérsic indices with $n_{870 \mu \mathrm{m}} \sim 1$ than the optical profiles with $n_{\mathrm{F} 160 \mathrm{~W}} \sim 2$. Figure 4(a) compares the FIR 

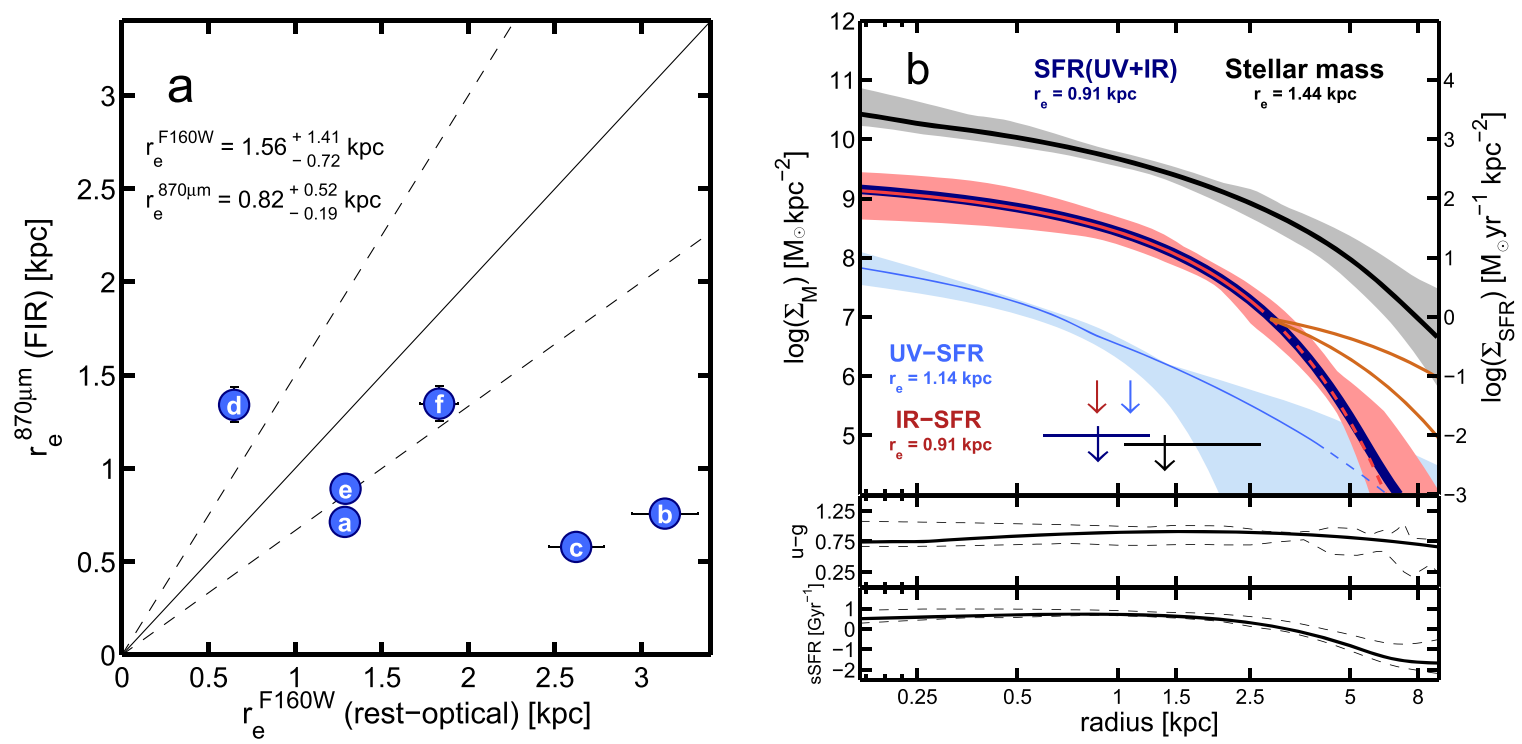

Figure 4. Left: comparison of the rest-frame optical (WFC3/F160W) and FIR (ALMA $870 \mu \mathrm{m}$ ) effective radii of compact SFGs. The solid line indicates the 1:1 relation, the dashed lines show the $1.5 \times$ size ratios. The FIR sizes are $\sim 1.6 \times$ smaller than the optical sizes and they exhibit a tighter distribution around $r_{e} \sim 1 \mathrm{kpc}$. Right: mean deconvolved stellar mass (black line) and SFR (blue and red lines) density profiles of compact SFGs. The shaded regions indicate the $1 \sigma$ dispersion. The dashed lines indicate the Sérsic fit to SFR profile below the UV and FIR detection limits. The orange lines show possible IR-SFR profiles undetected by ALMA. The arrows show the mean effective radii and the horizontal bars indicate their lower/upper limits determined from the $\pm 1 \sigma$ profiles. The bottom panels show the $u-g$ color profile and the sSFR profile.

and optical $r_{\mathrm{e}}$ and shows no clear correlation. Furthermore, the FIR sizes exhibit a tighter size distribution that suggests a relatively homogeneous population of compact, dusty, starbursts confined to the nuclear regions. The small star-forming regions with integrated SFRs consistent with the SF-MS imply that the nuclear starbursts have $\left\langle\Sigma_{\mathrm{SFR}}\right\rangle=\mathrm{SFR} / \pi r_{\mathrm{e}}^{2}$ up to $25 \times$ larger than a typical SF-MS disk with $r_{\mathrm{e}, \mathrm{SFR}} \sim 5 \mathrm{kpc}$ (Nelson et al. 2015).

The remarkable compactness of the dust continuum emission is consistent with recent results on SMGs and other IR-bright galaxies which report small FIR sizes (Gaussian FHWM $\sim 0$ ". 12) compared to the typical rest-frame optical sizes of SFGs at $z \sim 2-3$ (Ikarashi et al. 2015; Simpson et al. 2015; Tadaki et al. 2015). Nonetheless, joint studies of both the UV- and IR-SFRs, and the stellar mass profiles are required to fully constrain the regions where stars are being formed and to understand their role in the structural evolution of SFGs.

\subsection{Stellar mass, UV-SFR, and IR-SFR Surface Density Profiles}

Figure 4(b) shows the average stellar mass and SFR profiles of the compact SFGs as computed from their deconvolved, surface density profiles. The UV-SFR and IR-SFR profiles are determined by scaling the rest-frame luminosity profiles, probed by ACS/F850LP and ALMA $870 \mu \mathrm{m}$, to an integrated SFR using the conversion in Equation (1). The stellar mass profiles are determined from the rest-frame optical luminosity probed by WFC3/F160W (approximately g-band, $L_{\mathrm{g}}$ ) by using an empirical correlation between the stellar mass-to-light ratio, $M_{\star} / L_{\mathrm{g}}$, and the rest-frame $(u-g)$ color as determined from F125W and F160W (see, e.g., Szomoru et al. 2012). We account for resolution effects in the color profile by using the best-fit GALFIT models of the F125W and F160W brightness profiles.
The average (UV+IR) SFR profile is approximately $1.5 \times$ more concentrated than the average stellar mass profile and it is strongly dominated by the IR emission (UV/IR $\gtrsim 100)$ up to $r \sim 5 \mathrm{kpc}$. The specific SFR (sSFR $=\mathrm{SFR} / M$ ) is highest at $r \lesssim 2.5 \mathrm{kpc}$ and thus imply that most of the stellar mass growth is taking place within the inner few kiloparsecs of the galaxy. At $r \gtrsim 5 \mathrm{kpc}$, the sSFR is $\sim 100 \times$ lower thus indicating that the SFR has an almost negligible contribution to the stellar mass growth at large radii.

Note that the UV- and IR-SFR profiles are detected only up to $r \sim 3-4 \mathrm{kpc}\left(\Sigma_{\mathrm{SFR}} \sim 1 M_{\odot} \mathrm{kpc}^{-2}\right.$ and $\left.\sim 0.1 M_{\odot} \mathrm{kpc}^{-2}\right)$. Therefore, the results at larger radii are based on the best-fit Sérsic profiles. Nonetheless, we verify that even if the IR-SFR profiles had secondary components undetected by ALMA with $\Sigma_{\mathrm{SFR}}(r=8 \mathrm{kpc}) \sim 10 \times$ and $100 \times$ lower than the detection limit (orange lines in Figure 4(b)), the $r_{\mathrm{e}}$ would only increase by $\sim 5 \%$ and $20 \%$ and thus the SFR would still be more concentrated than the stellar mass.

\section{DISCUSSION}

In a simplified picture of galaxy growth, the average structural evolution of SFGs proceeds roughly along their well-defined scaling relations (blue arrows in Figure 5; e.g., van Dokkum et al. 2015; Barro et al. 2016). In this picture, massive compact quiescent galaxies at $z \sim 2$ would be descendants of smaller SFGs at higher- $z$ that achieve such high stellar densities by continuously growing in stellar mass and size fueled by extended SFR profiles. Alternatively, these SFGs could deviate from the smooth track due to dissipative processes that would rapidly increase their concentrations and potentially decrease their half-mass radii in strong nuclear starbursts (Dekel \& Burkert 2014; Wellons et al. 2015). The secular and dissipation-driven scenarios are not mutually exclusive. However, we aim to understand whether the massive dense cores of compact quiescent galaxies are primarily formed in dissipative processes. 

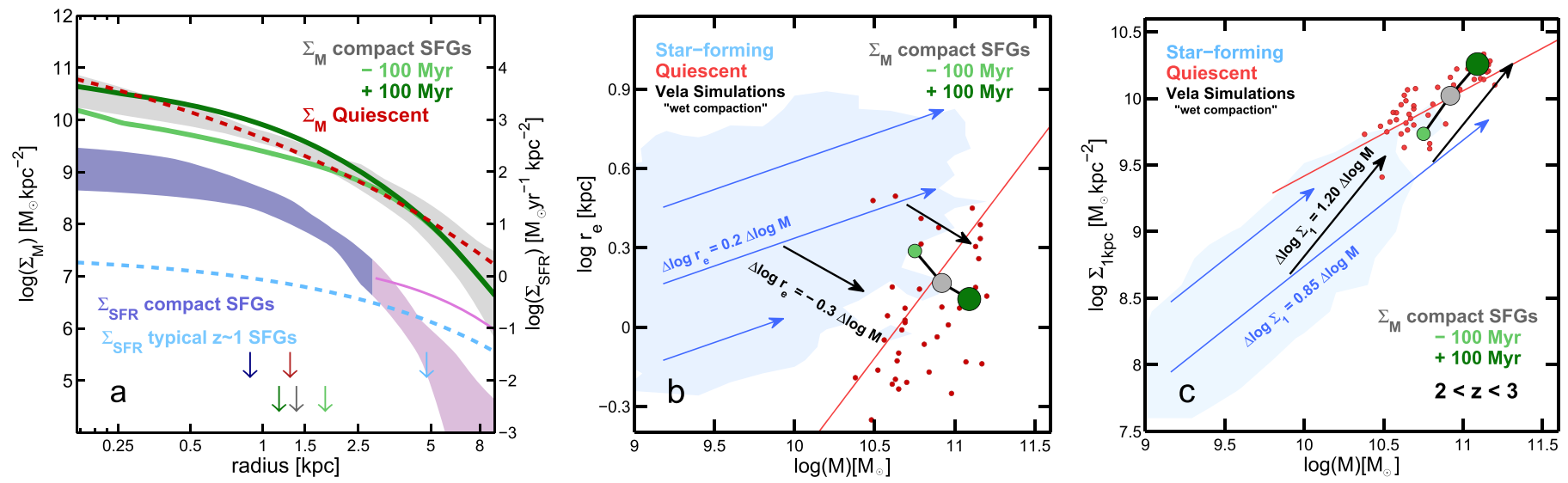

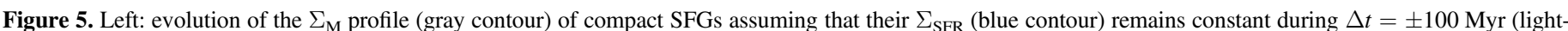

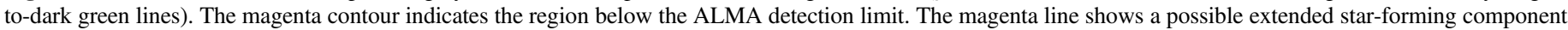

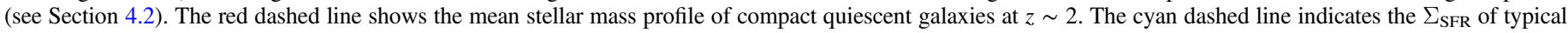

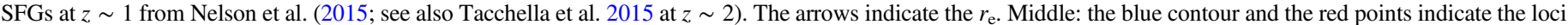

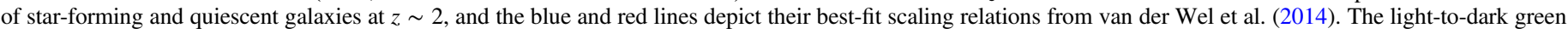

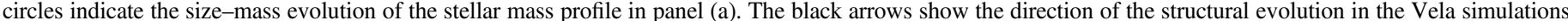

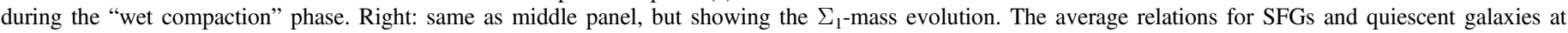
$z \sim 2$ are from Barro et al. (2016).

The strong nuclear starbursts embedded in larger stellar mass profiles found in compact SFGs are indeed an excellent match to the dissipation-driven scenario. The light-to-dark green lines and circles in Figure 5 show the predicted change in the stellar mass profile and the evolutionary tracks in $r_{\mathrm{e}}$ and central mass density for compact SFGs due to star formation, assuming that their SFR profiles remain constant during $\Delta t=200 \mathrm{Myr}$ (approximately $t_{\mathrm{dpl}}$ ). The significant stellar mass growth within the inner $r \lesssim 2 \mathrm{kpc}$ decreases the half-mass-radius by $16 \times$ from $r_{\text {e,mass }}=1.9$ to $1.2 \mathrm{kpc}$, while the central density within $r \leqslant 1 \mathrm{kpc}$ increases by $\sim 4 \times$ from $\log \left(\Sigma_{1}\right)=9.7$ to $10.3 M_{\odot} \mathrm{kpc}^{-2}$. If compact SFGs had more extended star formation at $r \gtrsim 3 \mathrm{kpc}$ the evolution of $\Sigma_{1}$ would be the same, while $r_{\text {e,mass }}$ would decrease less (e.g., $\sim 7 \%$ for the magenta line).

These evolutionary tracks are very similar to the predictions of the Vela simulations during the "wet compaction" phase (black arrows; e.g., Zolotov et al. 2015; Tacchella et al. 2016) and contrast with the expected evolution for typical SF-MS galaxies, which have extended SFR profiles with $\sim 100 \times$ lower central $\Sigma_{\text {SFR }}$ (cyan line in Figure 5(a)) and thus favor a more gradual increase of $\Sigma_{1}$ and a positive size evolution.

The short depletion times of compact SFGs and the similarity with the mean stellar mass profile of quiescent galaxies at $z \sim 2$ (red dashed line in Figure 5(a)) suggest that the nuclear starburst is unlikely to continue for more than a few hundred Myr, either because no further gas is accreted into the galaxy center or because the dense stellar component stabilizes the gas to prevent further star formation and eventually leads to galaxy quenching. This scenario is consistent with previous results indicating that the formation of a dense core precedes the shut down of star formation (e.g., Cheung et al. 2012; van Dokkum et al. 2014), and suggests that, at high redshift, both quenching and the dense cores are simultaneous consequences of enhanced periods of nuclear star formation that cause a rapid depletion of the gas reservoirs.

G.B. and M.K. acknowledge support from HST-AR-12847 and the Hellman Fellows Fund. P.G.P.-G. acknowledges support from grant AYA2015-70815-ERC. W.R. is supported by a CUniverse Grant (CUAASC) from Chulalongkorn University. This paper makes use of the following ALMA data: ADS/JAO.ALMA\#2013.1.00576.S. ALMA is a partnership of ESO (representing its member states), NSF (USA) and NINS (Japan), together with NRC (Canada), NSC and ASIAA (Taiwan), and KASI (Republic of Korea), in cooperation with the Republic of Chile. The Joint ALMA Observatory is operated by ESO, AUI/NRAO and NAOJ. The National Radio Astronomy Observatory is a facility of the National Science Foundation operated under cooperative agreement by Associated Universities, Inc.

\section{REFERENCES}

Barro, G., Faber, S. M., Koo, D. C., et al. 2016, ApJ, submitted (arXiv:1509. 00469)

Barro, G., Faber, S. M., Pérez-González, P. G., et al. 2013, ApJ, 765, 104

Barro, G., Faber, S. M., Pérez-González, P. G., et al. 2014, ApJ, 791, 52

Bell, E. F., Papovich, C., Wolf, C., et al. 2005, ApJ, 625, 23

Bruzual, G., \& Charlot, S. 2003, MNRAS, 344, 1000

Calzetti, D., Armus, L., Bohlin, R. C., et al. 2000, ApJ, 533, 682

Chabrier, G. 2003, PASP, 115, 763

Chary, R., \& Elbaz, D. 2001, ApJ, 556, 562

Cheung, E., Faber, S. M., Koo, D. C., et al. 2012, ApJ, 760, 131

Dale, D. A., \& Helou, G. 2002, ApJ, 576, 159

Dekel, A., \& Burkert, A. 2014, MNRAS, 438, 1870

Dekel, A., Sari, R., \& Ceverino, D. 2009, ApJ, 703, 785

Draine, B. T., \& Li, A. 2007, ApJ, 657, 810

Elbaz, D., Dickinson, M., Hwang, H. S., et al. 2011, A\&A, 533, A119

Fall, S. M., \& Efstathiou, G. 1980, MNRAS, 193, 189

Foreman-Mackey, D., Hogg, D. W., Lang, D., \& Goodman, J. 2013, PASP, 125,306

Grogin, N. A., Kocevski, D. D., Faber, S. M., et al. 2011, ApJS, 197, 35

Guo, Y., Ferguson, H. C., Giavalisco, M., et al. 2013, ApJS, 207, 24

Hopkins, P. F., Hernquist, L., Cox, T. J., \& Kereš, D. 2008, ApJS, 175, 356

Ikarashi, S., Ivison, R. J., Caputi, K. I., et al. 2015, ApJ, 810, 133

Kennicutt, R. C., Jr. 1998, ARA\&A, 36, 189

Kriek, M., van Dokkum, P. G., Labbé, I., et al. 2009, ApJ, 700, 221

Krist, J. 1995, in ASP Conf. Ser. 77, Astronomical Data Analysis Software and Systems IV, ed. R. A. Shaw, H. E. Payne, \& J. J. E. Hayes (San Francisco, CA: ASP), 349

Magdis, G. E., Daddi, E., Béthermin, M., et al. 2012, ApJ, 760, 6

Magnelli, B., Popesso, P., Berta, S., et al. 2013, A\&A, 553, A132

Mo, H. J., Mao, S., \& White, S. D. M. 1998, MNRAS, 295, 319 
Nelson, E. J., van Dokkum, P. G., Förster Schreiber, N. M., et al. 2015, ApJ, submitted (arXiv:1507.03999)

Nelson, E. J., van Dokkum, P. G., Momcheva, I., et al. 2013, ApJL, 763, L16 Noeske, K. G., Weiner, B. J., Faber, S. M., et al. 2007, ApJL, 660, L43

Peng, C. Y., Ho, L. C., Impey, C. D., \& Rix, H.-W. 2002, AJ, 124, 266

Pérez-González, P. G., Trujillo, I., Barro, G., et al. 2008, ApJ, 687, 50

Rieke, G. H., Alonso-Herrero, A., Weiner, B. J., et al. 2009, ApJ, 692, 556

Sandstrom, K. M., Leroy, A. K., Walter, F., et al. 2013, ApJ, 777, 5

Santini, P., Ferguson, H. C., Fontana, A., et al. 2015, ApJ, 801, 97

Simpson, J. M., Smail, I., Swinbank, A. M., et al. 2015, ApJ, 807, 128

Steidel, C. C., Rudie, G. C., Strom, A. L., et al. 2014, ApJ, 795, 165

Szomoru, D., Franx, M., \& van Dokkum, P. G. 2012, ApJ, 749, 121

Tacchella, S., Carollo, C. M., Renzini, A., et al. 2015, Sci, 348, 314

Tacchella, S., Dekel, A., Carollo, C. M., et al. 2016, MNRAS, 458, 242
Tacconi, L. J., Genzel, R., Neri, R., et al. 2010, Natur, 463, 781

Tadaki, K.-i., Kohno, K., Kodama, T., et al. 2015, ApJL, 811, L3

van der Wel, A., Bell, E. F., Häussler, B., et al. 2012, ApJS, 203, 24

van der Wel, A., Franx, M., van Dokkum, P. G., et al. 2014, ApJ, 788, 28

van Dokkum, P. G., Bezanson, R., van der Wel, A., et al. 2014, ApJ, 791, 45

van Dokkum, P. G., Nelson, E. J., Franx, M., et al. 2015, ApJ, 813, 23

Wellons, S., Torrey, P., Ma, C.-P., et al. 2015, MNRAS, 449, 361

Whitaker, K. E., van Dokkum, P. G., Brammer, G., \& Franx, M. 2012, ApJL, 754, L29

Wuyts, S., Förster Schreiber, N. M., Genzel, R., et al. 2012, ApJ, 753, 114

Wuyts, S., Förster Schreiber, N. M., Lutz, D., et al. 2011, ApJ, 738, 106

Wuyts, S., Förster Schreiber, N. M., Nelson, E. J., et al. 2013, ApJ, 779,135

Zolotov, A., Dekel, A., Mandelker, N., et al. 2015, MNRAS, 450, 2327 